\title{
Association of obstructive sleep apnea with non-alcoholic fatty liver disease in patients with obesity: an observational study
}

\author{
Silvia Bettini ${ }^{1}$ - Roberto Serra ${ }^{2} \cdot$ Roberto Fabris $^{2} \cdot$ Chiara Dal Prà $^{2} \cdot$ Francesca Favaretto $^{1,2} \cdot$ Francesca Dassie $^{1}$. \\ Claudio Duso ${ }^{1} \cdot$ Roberto Vettor $^{1,2} \cdot$ Luca Busetto $^{1,2}$
}

Received: 18 November 2020 / Revised: 6 January 2021 / Accepted: 7 January 2021 / Published online: 3 April 2021

(c) The Author(s) 2021

\begin{abstract}
Purpose Obstructive Sleep Apnea (OSA) is associated with the presence and severity of Non-Alcoholic Fatty Liver Disease (NAFLD). We aimed to investigate the relationship between the severity of OSA and NAFLD and to recognize a polysomnographic parameter correlated with progression of fibrosis, determined by a non-invasive score of liver fibrosis, FIBrosis-4 index (FIB-4), in patients affected by severe obesity and OSA.

Methods We enrolled 334 patients (Body Mass Index, BMI $44.78 \pm 8.99 \mathrm{~kg} / \mathrm{m}^{2}$ ), divided into classes according to severity of OSA evaluated with Apnea Hypopnea Index (AHI): OSAS 0 or absent (17\%), mild OSA (26\%), moderate OSA (20\%), severe OSAS (37\%). We studied anthropometric, polysomnographic, biochemical data and FIB-4. A multiple regression model was computed to identify a polysomnographic independent predictor of FIB-4 among those parameters previously simple correlated with FIB-4.

Results The severity of OSA was associated with a decrease in High-Density Lipoprotein-cholesterol (HDL) and an increase in BMI, triglycerides, Homeostasis model assessment insulin-resistance index (HOMA), transaminases and FIB-4. FIB-4 correlated with sex, age, BMI, AHI, mean percentage oxyhaemoglobin (meanSaO2\%), number of desaturations, platelets, transaminases, HDL, triglycerides and HOMA. The only variables independently related to FIB-4 were sex, BMI, triglycerides and meanSpO2 ( $r=0.47$, AdjRsqr $=0.197)$.

Conclusion MeanSpO2\% represented an independent determinant for the worsening of FIB-4 in patients with severe obesity and OSA. Hence, it could hypothesize a clinical role of meanSaO2\% in recognizing patients with obesity and OSA and higher risk of developing advanced fibrosis and, thus, to undergo further investigation.
\end{abstract}

Level III Evidence obtained from well-designed cohort analytic studies.

Keywords Obstructive Sleep Apnea Syndrome $\cdot$ Non-Alcoholic Fatty Liver Disease $\cdot$ FIB-4 · Obesity · Fibrosis

\section{Introduction}

In the last decades we observed a pandemic increase in the prevalence of metabolic diseases such as obesity, Type 2 Diabetes Mellitus (T2DM) and non-alcoholic fatty liver disease (NAFLD) [1-3] and, on the other hand, of Obstructive Sleep Apnea (OSA) [4-6]. NAFLD is an emerging disease in western countries, representing about $75 \%$ of all chronic

Silvia Bettini

d.ssa.silvia.bettini@gmail.com

1 Department of Medicine, Internal Medicine 3, University of Padova, Via Giustiniani 2, 35128 Padova, Italy

2 Center for the Study and the Integrated Management of Obesity, Padova University Hospital, Padova, Italy liver diseases and one of the most worrying complications of obesity. NAFLD is associated with progressive insulinresistance [1-3] and, on the other hand, insulin-resistance predicts NAFLD improvement in patients undergoing a dietary intervention for weight loss [7]. NAFLD includes different clinical conditions: Non-Alcoholic Fatty Liver (NAFL), histologically characterized by simple liver steatosis, and Non-Alcoholic Steato-Hepatitis (NASH) that covers a broad spectrum of complications, from fibrosis to cirrhosis to hepatocellular carcinoma $[2,3]$. It was estimated that the global prevalence of NAFLD is around 25\% [1] and that it grows consensually with related metabolic diseases, in particular obesity $[2,3]$. Indeed, several genetic modifiers of NAFLD have been identified and the best-characterized 
genetic association is with PNPLA3, found also in patients without insulin-resistance [2].

OSA is characterized by repetitive episodes of partial or total loss of respiratory airflow during sleep with the collapse of the upper airway during inspiration and is accompanied by strenuous breathing [4]. In general population, OSA prevalence is approximately $10-17 \%$ in men and 3-9\% in women [6], whereas the prevalence of OSA in people with obesity is about $48 \%$ in men and $38 \%$ in women [8]. Visceral obesity is related to OSA presence and severity $[9,10]$ and OSA is associated with an increased cardiovascular risk, probably driven by changes in glucose and lipid metabolism [11]. Pathophysiological mechanisms by which obesity promotes OSA are not only a simplistic mechanical impact that the expansion of adipose tissue has on the airways. Thus, it has been suggested that the expansion of the visceral adipose tissue, the alteration of the adipocytokines, the increase in circulatory levels of inflammatory mediators such as Tumor Necrosis Factor (TNF) $\alpha$, interleukin (IL)-6, IL-1, IL-1 $\beta$ and nuclear factor $\mathrm{kB}(\mathrm{NF}-\mathrm{kB})$ and the recruitment of immune cells determine a chronic low-grade inflammation which is implicated in the appearance and aggravation of OSA [9, 11-17]. The association between obesity and lung disease is not limited to OSA. Covid-19 respiratory syndrome is a current example of how adipose tissue disfunction can lead to lung injury [18].

In the same way, NAFLD positively correlates with visceral fat depot and obesity comorbidities (such as T2DM and OSA) worsen the histopathological features of NAFLD [2, $3,19]$. As for OSA, adipocytokines play an important role in the pathogenesis and progression of NAFLD, together with a direct effect of insulin resistance as well as oxidative stress [20-24].

Identifying patients with the greatest risk of evolution of NAFLD to NASH represents a challenge for clinicians. It is worth to note that fibrosis is the predictive criterion for a poor prognosis $[2,3,25,26]$ and, although liver biopsy is considered the diagnostic gold standard to detect fibrosis, it is expensive, invasive and therefore not suitable for general population studies [27]. Indeed, liver biopsy is also affected by sampling bias, providing only a very small sample with the risk of not to be representative for the amount of hepatic fibrosis in the whole liver [28]. Thus, it is recommended to calculate non-invasive fibrosis biomarkers and scores in patients with NAFLD, to identify patients at higher risk to undergo to liver biopsy [2, 3]. Among these, FIBrosis-4 index (FIB-4), NAFLD fibrosis scores, alanine aminotransferase (ALT)/aspartate aminotransferase (AST) ratio, Aspartate aminotransferases-to-Platelet Ratio Index (APRI) have been validated under several conditions of chronic liver disease and in the NAFLD, and FIB-4 especially, demonstrating sufficient sensitivity and specificity to be used in clinical practice [26, 29-31].
A growing amount of evidence has correlated OSA with the presence of NAFLD and it has been demonstrated a direct association between the severity of the former and the progression of the latter [32]. Thus, OSA patients should be screened for the presence and severity of NAFLD [32]. Non-invasive biomarkers and scores could become a screening tool to identify patients to be referred to liver biopsy and therefore to prevent NAFLD progression in patients living with obesity and OSA. It was suggested that the chronic intermittent hypoxia present in OSA triggers and increases the accumulation of triglycerides (TG), low-grade inflammation, tissue necrosis and liver fibrosis [24, 33]. However, pathophysiological mechanisms by which intermittent hypoxia lead to simple liver steatosis to the development of fibrosis and cirrhosis are still unclear. Therefore, the aim of this study was firstly, to analyze the association between OSA and NAFLD in patients with severe obesity and to investigate a possible direct correlation between polysomnographic parameters and a non-invasive score of liver fibrosis, and secondly to recognize an independent determinant of OSA to predict NAFLD progression.

\section{Material and methods}

\section{Patients}

Three hundred and thirty-four Caucasian patients with obesity (Boby Mass Index, BMI $\geq 30 \mathrm{~kg} / \mathrm{m}^{2}$ ) were enrolled at the Centre for the Study and Integrated Treatment of Obesity, Padua University Hospital, in the period 2013-2019. Patients underwent a multi-disciplinary evaluation according to a standard clinical protocol and a complete medical history was taken regarding eating, physical activity, smoking and drinking habits, drugs, past and current medical conditions. Indeed, these patients underwent to polysomnography according to the suspect of OSA on the basis of clinical evaluation and a validated questionnaire (the Epworth Sleepiness Scale, ESS). These subjects were also eligible for bariatric surgery and, thus, provided the result of a simple abdominal ultrasound scan (US), as pre-surgery exam according to a standard clinical protocol. All US confirmed the presence of fatty liver. Specific exclusion criteria for this study were previous bariatric surgery, continuous positive airway pressure therapy (CPAP), unavailability of all clinical anamnestic, anthropometric, biochemical and polysomnographic parameters, excessive alcohol consumption (daily alcohol consumption $\geq 30 \mathrm{~g}$ for men and $\geq 20 \mathrm{~g}$ for women [2]), non-metabolic liver disease (i.e. viral or autoimmune hepatitis, genetic hemochromatosis, Wilson disease), a current infection and use of hepatotoxic drugs. Laboratory exams 
and polysomnography were scheduled before bariatric surgery closely in time not greater than two months, and with not significant difference in weight.

All anthropometric measurements were taken with subjects wearing only light clothes without shoes. Height was measured to the nearest $0.01 \mathrm{~m}$. Body weight was determined to the nearest $0.1 \mathrm{~kg}$ using a calibrated balance beam scale.

All subjects gave written informed consent in accordance with the Declaration of Helsinki. The protocol was approved by the 'Padua Ethical Committee for Clinical Research' (2892P, 10/06/2013).

\section{Biochemical assessment}

For each patient, all blood tests were performed in the morning, after $8 \mathrm{~h}$ of fasting. All biochemical blood analyses have been performed with standard diagnostic kit according to WHO First International Reference Standard: fasting plasma glucose (FPG) (Glucose HK Gen.3, Roche Diagnostic, Indianapolis, IN, USA), basal insulin, IL-6, TNF $\alpha$ (IMMULITE 2000 Immunoassay, Siemens Healthcare GmbH, Erlangen, Germany), high-sensitivity C-Reactive Protein (hs-CRP) (CardioPhase High Sensitivity C-Reactive Protein, Siemens Healthcare, Erlangen, Germany), Leptin (Leptin-RIA-CT, Mediagnost, Reutlingen, Germany), vitamin D3 (Diasorin Liaison XL LAS, Saluggia, Italy). Platelets were measured by flow cytometry (Sysmex Europe GmbH, Norderstedt, Germany), lipid profile [(total cholesterol (TC), High-Density Lipoprotein-cholesterol (HDL-cholesterol) and TG] by spectrophotometer (Cobas 8000, Roche Diagnostic, Indianapolis, IN, USA). Low Density Lipoprotein-cholesterol (LDL-cholesterol) was calculated according to Friedewald [34]. ALT, AST, gamma glutamyltransferase (GGT) levels were assayed by enzymatic method with the addition of pyridoxal-5-phosphate in compliance with IFCC reference methods [35]. Leptin, IL-6 and TNF- $\alpha$ were not available for 63 patients and hs-CRP for 124 patients. Missing data were homogeneously distributed into OSA categories.

The insulin-resistance index was indirectly estimated using the homeostasis model assessment (HOMA) as follow: [fasting serum insulin $(\mu \mathrm{U} / \mathrm{ml}) \times$ fasting plasma glucose $(\mathrm{mmol} / \mathrm{l})] / 22.5[36]$.

\section{Non-invasive fibrosis score}

FIB-4 was calculated using the following formula: [age (years) $\times$ AST]/[platelet counts $\left(\times 10^{9} / 1\right) \times$ ALT $\left.^{1 / 2}\right][26,30]$. A low cutoff point $(<1.30)$ and a high cutoff point $(>2.67)$ best discriminate between the absence and presence of advanced fibrosis, respectively [30].

\section{Polysomnographic evaluation}

The polysomnographic analysis was performed using the SOMNOtouchTM ${ }^{\circledR}$ NIBP device (SOMNOmedics Italia, Bellusco, Italy) and a complete cardiorespiratory night monitoring was carried out for at least $8 \mathrm{~h}$. During polysomnography peripheral, blood oxygen saturation, respiratory flow in the upper airway, respiratory movements of the chest and abdomen, snoring phases, the position of the patient, blood pressure and electrocardiogram with acquisition of peripheral signals were recorded. The recorded layout and thus the criterion for assigning hypopneas scoring was automatically analyzed by the DOMINO software ${ }^{\circledR}$, validated by SOMNOmedics, and subsequently it was manually validated by an expert operator according to the most recent American Academy of Sleep Medicine (AASM) criteria [37]. The anthropometric data included age, height and weight. The polysomnographic data encompassed Apnea Hypopnea Index (AHI), minimum percentage oxyhaemoglobin saturation $(\mathrm{mSaO} 2 \%)$, mean percentage oxyhaemoglobin saturation (meanSaO2\%), percentage of time with oxyhaemoglobin saturation percentage less than 90 (TS $<90 \%$ ) and number of desaturations with oxyhaemoglobin saturation $<90 \%(\mathrm{nSaO} 2<90 \%)$.

\section{Statistical analysis}

Statistical analyses were performed using the SigmaPlot v.14 (Systat Software, Adalta, Arezzo, Italy). All variables were tested by normal Test (Shapiro-Wilk test) and Equal Variance Test (Brown-Forsythe). One Way Analysis of Variance was used when Normality Test and Equal Variance Test have been passed (data are presented as mean values \pm standard deviations) or, if not, with the Kruskal-Wallis One Way Analysis of Variance on Ranks (data are presented as median value (25th-75th percentile)). Chi-square test was carried out for categorical variables. Pearson's correlation coefficient $(r)$ and the relative $p$ values were calculated to analyze simple linear correlations between two variables. A multiple regression model with adjustment for presence of diabetes, hypertension and dyslipidemia was computed with FIB-4 as the dependent variable and the variables found to be simple correlated with FIB-4 as independent variables. Variant Inflation Factor (VIF) was considered as measure of multicollinearity and a value greater than 4 was considered as cut off for excluding variables from the analysis. In all analyses, the $p$ values were two-sided and a $p$ value lower than 0.05 was considered statistically significant. 


\section{Results}

Three hundred and thirty-four patients with severe obesity had a mean age of $48 \pm 12$ years (range 18-79), 158 were male (47\%) and the mean BMI was $44.78 \pm 8.99 \mathrm{~kg} /$ $\mathrm{m}^{2}$ (IQR 38.5-49.9). Patients with hypertension were 204 (61.1\%); dyslipidemia, defined according to recent guidelines [38], was present in 197 patients (59\%). According to the American Diabetes Association [39], 101 patients were affected by T2DM (30.2\%), 54 (16.2\%) had prediabetes (impaired fasting glycemia or/and impaired glucose tolerance at the oral glucose tolerance test), 179 (53.6\%) had a normal glycemia. Clinical, laboratory and polysomnographic evaluations of the 334 patients, divided in relation to OSA severity (AHI), are reported in Table 1. Fifty seven $(17 \%)$ of the patients were classified as no OSA $(\mathrm{AHI}<5), 88(26 \%)$ as mild OSA (AHI 5-14), $65(20 \%)$ as moderate OSA (AHI 15-29) and 124 (37\%) as severe OSA $(\mathrm{AHI} \geq 30)$. As expected, all polysomnographic parameters

Table 1 Anthropometric characteristics, comorbidities, polysomnographic parameters, biochemical data and liver fibrosis score in 334 patients with obesity, divided according to OSA severity

\begin{tabular}{|c|c|c|c|c|c|c|}
\hline & & $\begin{array}{l}\text { No OSA } \\
(n=57)\end{array}$ & $\begin{array}{l}\text { Mild OSA } \\
(n=88)\end{array}$ & $\begin{array}{l}\text { Moderate OSA } \\
(n=65)\end{array}$ & $\begin{array}{l}\text { Severe OSA } \\
(n=124)\end{array}$ & $P$ \\
\hline \multirow[t]{4}{*}{ Anthropometric data } & $\operatorname{Sex}(M / F)$ & $12 / 45$ & $28 / 60$ & $33 / 32$ & $85 / 39$ & $<0.001$ \\
\hline & Age (y) & $41.1 \pm 14.1$ & $48.7 \pm 10.6$ & $50.2 \pm 10.8$ & $49.8 \pm 12.2$ & $<0.001$ \\
\hline & Weight (Kg) & $114(100.4-130.6)$ & $115.8(102.2-133)$ & $122.9(108-150.4)$ & $132(116.2-154.8)$ & $<0.001$ \\
\hline & BMI $\left(\mathrm{Kg} / \mathrm{m}^{2}\right)$ & $40.7(36-47.5)$ & $41(37.4-47.9)$ & $44.3(40.1-49.2)$ & $45.2(39.6-51.2)$ & $<0.001$ \\
\hline \multirow[t]{4}{*}{ Comorbidities } & $\mathrm{T} 2 \mathrm{DM}$ & $10(17.5)$ & $25(28.4)$ & $23(35.4)$ & $43(34.7)$ & 0.079 \\
\hline & Prediabetes & $8(14)$ & $18(20.4)$ & $10(15.4)$ & $18(15.3)$ & 0.645 \\
\hline & Hypertension & $21(36.8)$ & $50(56.8)$ & $39(60)$ & $94(75.8)$ & $<0.001$ \\
\hline & Dyslipidemia & $23(40.3)$ & $53(60.2)$ & $43(66.1)$ & $78(62.9)$ & 0.015 \\
\hline \multirow[t]{4}{*}{ Polysomnographic data } & $\mathrm{mSaO} 2 \%$ & $87(81-91)$ & $84(77-87)$ & $78(69-83)$ & $68(61-76)$ & $<0.001$ \\
\hline & meanSaO2\% & $95(94-96)$ & $94(93-95)$ & $93(92-95)$ & $92(89-93)$ & $<0.001$ \\
\hline & $\mathrm{nSaO} 2<90 \%$ & $0.1(0.0-0.6)$ & $1.4(0.5-4.175)$ & $6(2.60-9.30)$ & $25.1(12.2-19.6)$ & $<0.001$ \\
\hline & $\mathrm{TS}<90 \%$ & $0.1(0.0-1.6)$ & $1.2(0.3-5.1)$ & $5.2(1.7-13.8)$ & $30.6(12.3-48.2)$ & $<0.001$ \\
\hline \multirow[t]{16}{*}{ Biochemical data } & $\mathrm{TC}(\mathrm{mg} / \mathrm{dL})$ & $175(152-190)$ & $187(156-209)$ & $187(163-221)$ & $176(154-206)$ & 0.187 \\
\hline & LDL (mg/dL) & $99(89-128)$ & $118(87-138)$ & $113(96-138)$ & $113(89-138)$ & 0.152 \\
\hline & HDL (mg/dL) & $49(38-56)$ & $46(39-57)$ & $42(37-51)$ & $41(34-48)$ & $<0.001$ \\
\hline & $\mathrm{TG}(\mathrm{mg} / \mathrm{dL})$ & $106(67-133)$ & $103(73-133)$ & $120(95-197)$ & $120(87-165)$ & 0.008 \\
\hline & Vitamin D3 (nmol/L) & $36(22-52)$ & $35(23-54)$ & $27(19-42)$ & $27(18-40)$ & 0.012 \\
\hline & FPG $(\mathrm{mmol} / \mathrm{L})$ & $5.2(5-5.8)$ & $5.7(5 .-6.6)$ & $5.8(5.1-7.3)$ & $5.8(5.3-7)$ & $<0.001$ \\
\hline & Insulin (mU/L) & $12.7(8.5-24)$ & $14.3(9.6-22.5)$ & $20(13-30)$ & $22.2(16-36)$ & $<0.001$ \\
\hline & HOMA & $2.8(1.8-5.8)$ & $4(2.3-5.8)$ & $5.3(3.1-8.8)$ & $6(4-8.9)$ & $<0.001$ \\
\hline & Platelets $\left(\times 10^{9} / \mathrm{L}\right)$ & $257(213-311)$ & $236(211-295)$ & $242(208-306)$ & $238(190-269)$ & 0.042 \\
\hline & $\operatorname{ALT}(\mathrm{U} / \mathrm{L})$ & $23(16-31)$ & $23(18-37)$ & $26(20-33)$ & $29(21-40)$ & 0.016 \\
\hline & AST (U/L) & $23(18-32)$ & $23(19-32)$ & $26(21-32)$ & $30(24-42)$ & $<0.001$ \\
\hline & GGT (U/L) & $21(14-37)$ & $21(14-40)$ & $29(18-44)$ & $30(22-44)$ & $<0.001$ \\
\hline & Leptin (ug/L) & $33(22-48)$ & $36(20-50)$ & $34(21-47)$ & $31(19-44)$ & 0.661 \\
\hline & hs-CRP (mg/L) & $3.94(1.95-6.88)$ & $4.36(2.41-7.14)$ & $4.14(2.91-7.65)$ & $4.72(3.24-6.73)$ & 0.912 \\
\hline & IL-6 (ng/L) & $1.9(1.9-3.28)$ & $2.1(1.9-3.1)$ & $2.4(1.9-4.1)$ & $2.6(1.9-3.9)$ & 0.024 \\
\hline & TNF- $\alpha(n g / L)$ & $7.1(5.85-8.6)$ & $6.8(5.6-9.5)$ & $8(6.42-9.4)$ & $7.7(6.18-9.35)$ & 0.133 \\
\hline Liver fibrosis score & FIB-4 & $0.79(0.48-1.26)$ & $0.97(073-1.42)$ & $1.07(0.74-1.48)$ & $1.20(0.91-1.85)$ & $<0.001$ \\
\hline
\end{tabular}

$M$ male, $F$ female, $B M I$ Body Mass Index, T2DM Type 2 Diabetes Mellitus, $\mathrm{mSaO} \%$ minimum percentage oxyhaemoglobin saturation (SaO2) $\%, n \mathrm{SaO} 2<90 \%$ number of desaturations with a SaO2 $<90 \%, T S<90 \%$ the percentage of time with $\mathrm{SaO} 2<90 \%, T C$ total cholesterol, $L D L \mathrm{Low}$ Density Lipoprotein-cholesterol, $H D L$ High-Density Lipoprotein-cholesterol, $T G$ triglycerides, FPG Fasting plasma glucose, HOMA Homeostasis model assessment-insulin-resistance index, $A L T$ alanine aminotransferase, $A S T$ aspartate aminotransferase, GGT gamma glutamyl transferase, $h s$-CRP high-sensitivity C-Reactive Protein, TNF- $a$ Tumor Necrosis Factor-a, IL-6 interleukin-6, FIB-4 Fibrosis-4 index

Statistical analysis between the four groups was performed with One Way Analysis of Variance when Normality Test (Shapiro-Wilk) and Equal Variance Test (Brown-Forsythe) have been passed (data are presented as mean values \pm standard deviations) or, if not, with the Kruskal-Wallis One Way Analysis of Variance on Ranks (data are presented as median value (25th-75th percentile)) and with Chi-square test in categorical variables; results were reported in the $\mathrm{p}$ column 
$(\mathrm{mSaO} 2 \%$, meanSaO2\%, $\mathrm{nSaO} 2<90 \%$ and $\mathrm{TS}<90 \%)$ changed significantly with the degree of OSA severity $(p<0.001)$. Particularly, patients with more severe OSA displayed a greater number of nocturnal desaturations, a lower average and minimum oxyhaemoglobin saturation values and they spent more time with saturation values less than $90 \%$.

The progression versus higher levels of OSA severity was associated with male $(p<0.001)$, age $(p<0.001)$, weight $(p<0.001)$, presence of hypertension $(p<0.001)$ and dyslipidemia $(p=0.015)$; even though not statistically significant, the percentage of patients with diabetes was gradually higher going through OSA classes. Consensually, the more severe OSA the higher were FPG $(p<0.001)$, insulin $(p<0.001)$, and HOMA $(p<0.001)$ levels. Moreover, HDL and TG levels significantly decreased $(p<0.001)$ and increased $(p=0.008)$, respectively, with OSA severity. We found that only IL-6 $(p=0.024)$ and not hs-CRP and TNF-a, was associated with OSA severity. Interestingly, also Vitamin D3 levels were statistically different between OSA classes.

Confirming the association between NAFLD and OSA severity, we showed a statistically significant increase of transaminase levels (ALT $p=0.016$, AST $p<0.001$, GGT $p<0.001)$ and a decrease of platelets $(p=0.042)$, with OSA severity. The relationship existing among the two diseases has been demonstrated by the association between AHI and the score of liver fibrosis, FIB-4 ( $p<0.001)$ (Fig. 1).

\section{Linear correlation analyses and multiple regression}

FIB-4 values were significantly correlated with sex (male $=0$, female $=1, r=-0.274, p<0.001)$, age $(r=0.416$, $p<0.001$ ), diabetes (presence $=1$, absence $=0, r=0.154$, $p<0.01)$, dyslipidemia $(r=0.179, p<0.01)$, hypertension $(r=0.208, p<0.001)$, AHI $(r=0.148, p<0.01)$; meanSaO2\% ( $r=-0.131, p<0.05) ; \mathrm{nSaO} 2<90 \%$ $(r=0.225, p<0.01)$, BMI $(-0.169, p<0.01)$, platelets $(r=-0.604, p<0.001)$, AST $(r=0.645, p<0.001)$, ALT $(r=0.341, p<0.001)$, GGT $(r=0.405, p<0.001)$, HDL $(r=-0.185, p<0.001), \mathrm{TG}(r=0.279, p<0.001)$, HOMA $(r=0.228,<0.001)$, leptin $(r=0.212, p<0.001)$ (Table 1 , simple linear coefficients $(r)$ ).

To investigate the role of different polysomnographic parameters as independent predictors of liver fibrosis, we computed a multiple regression model with FIB-4 as the dependent variable and the variables found to be significantly related to FIB-4 in simple correlation analysis as independent variables. We excluded some multicollinear variables (in particular, $\mathrm{AHI}$ and $\mathrm{nSaO} 2<90 \%$ because of presenting a VIF greater than 4), and transaminases, platelets and age because already included in the FIB-4 score. In this model, TG, sex, meanSaO2\%, BMI and HOMA were

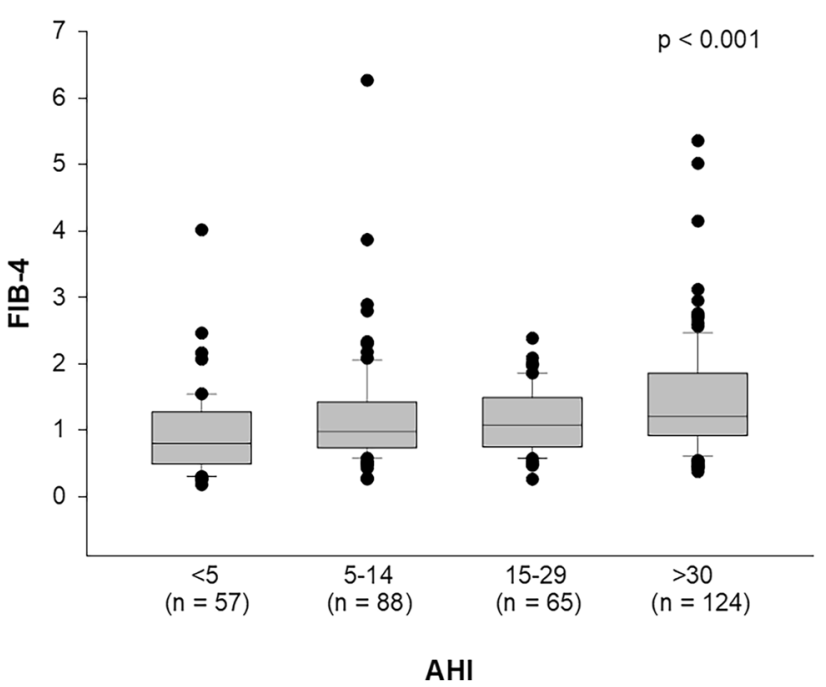

Fig. 1 Association of FIBrosis-4 index (FIB-4) with severity of Obstructive Sleep Apnea, determined by Apnea Hypopnea Index (AHI). Results are reported as box plot graphs: the box represents the lower and upper quartiles, the line in the box represents the median, the whiskers show the lowest and highest values, and the outliers are represented by black circles. Statistical analysis was performed by the Kruskal-Wallis One Way Analysis of Variance on Ranks

the only variables independently correlated with REE in 334 patients with obesity $(r=0.47$, AdjRsqr $=0.197)($ Table 2$)$.

\section{Discussion}

We have investigated the relationship between the presence and severity of OSA and liver fibrosis in a large sample of patients with severe obesity undergoing polysomnography for diagnostic reasons. Consensually with literature, OSA was associated with a greater severity of obesity both in terms of BMI and metabolic complications. We demonstrated an independent correlation between the mean $\mathrm{SaO} 2 \%$ values, an OSA severity index, and FIB-4 values, suggesting an independent role of this parameter in recognizing patients with greater risk of NAFLD progression towards stages characterized by growing fibrosis.

In our population the prevalence of hypertension, dyslipidemia and diabetes increased consensually with the severity of the OSA, with statistical significance for the two first, in agreement with previous studies $[4,5,11,40]$. In particular, AHI was associated with a reduction of HDL plasma levels and with a TG increase, both parameters typically involved in the metabolic syndrome and the latter in NAFLD, suggesting an association between hypoxia and lipids accumulation. Accordingly, we observed an increase in FPG, insulin levels and the HOMA index with the increase in AHI. We could hypothesize a direct relationship between the severity of insulin resistance and the worsening of the OSA, 
Table 2 Simple linear correlation and regression analyses of FIB-4 in 334 patients with obesity

\begin{tabular}{|c|c|c|c|}
\hline & \multicolumn{3}{|l|}{ FIB-4 } \\
\hline & $\begin{array}{l}\text { Simple linear } \\
\text { coefficients }(r)\end{array}$ & $\begin{array}{l}\text { Regression } \\
\text { coefficients }(\beta)\end{array}$ & Regression $p$ \\
\hline $\operatorname{Sex}^{\wedge}$ & $-0.274 * * *$ & -0.178 & 0.004 \\
\hline Age & $0.416^{* * *}$ & & \\
\hline Diabetes\# & $0.154 * *$ & & \\
\hline Dyslipidemia\# & $0.179 * *$ & & \\
\hline Hypertension\# & $0.208 * * *$ & & \\
\hline AHI & $0.148 * *$ & & \\
\hline meanSaO2\% & $-0.131^{*}$ & -0.157 & 0.016 \\
\hline $\mathrm{nSaO} 2<90 \%$ & $0.225 * *$ & & \\
\hline BMI & $-0.169 * *$ & -0.208 & 0.002 \\
\hline Platelets & $-0.604 * * *$ & & \\
\hline AST & $0.645^{* * *}$ & & \\
\hline ALT & $0.341 * * *$ & & \\
\hline GGT & $0.405 * * *$ & & \\
\hline HDL & $-0.185^{* * *}$ & & \\
\hline TG & $0.279 * * *$ & 0.218 & $<0.001$ \\
\hline HOMA & $0.228 * * *$ & 0.137 & 0.044 \\
\hline Leptin & $0.212 * * *$ & & \\
\hline
\end{tabular}

AHI Apnea Hypopnea Index, meanSaO2\% mean oxygen saturation $\%, n \mathrm{SaO} 2<90 \%$ number of desaturations with a $\mathrm{SaO} 2<90 \%, B M I$ Body Mass Index, AST aspartate aminotransferase, $A L T$ alanine aminotransferase, $G G T$ gamma glutamyl transferase, $H D L$ High-Density Lipoprotein-cholesterol, $T G$ triglycerides, HOMA Homeostasis model assessment insulin-resistance index, FIB-4 Fibrosis-4 index

Simple linear correlations were calculated by Pearson's correlation. Regression coefficient e Regression $p$ of the variables found to be independent related to FIB- 4 were reported. ${ }^{\wedge}$ male $=0$, female $=1$. \#absence $=0$, presence $=1$

$* p<0.05 ; * * p<0.01 ; * * * p<0.001$

independent of the degree of obesity, roughly determined by BMI. The treatment of OSA with CPAP is associated with an improvement in insulin resistance in the absence of significant changes in the BMI [41]. Moreover, the IL-6 values, a not specific marker of inflammation, were related to the severity of the OSA. These variations agree with the hypothesis that the low-grade inflammation plays an important pathogenetic role both in OSA-related cardiovascular complications and in the progression from fatty liver disease to NASH [33]. On the other hand, no correlation was demonstrated between IL- 6 and FIB-4, probably due to the lower plasma levels of IL-6 respect to the levels in the adipose tissue.

Focusing on transaminases levels, we noticed that they were similar in the group without OSA and with mild OSA; on the other hand, in the groups with more severe degrees of OSA, a pathological increase in transaminases has been reported. These data seemed to confirm an association between worsening AHI and NAFLD, although recent guidelines have underlined that transaminases have a poor predictive value for the evolution from NAFLD to NASH $[2,3]$. In patients with NAFLD, it is recommended to calculate non-invasive fibrosis markers and scores, to identify patients at higher risk to be referred to liver biopsy. In our study we have chosen to use FIB-4 whose sensitivity and specificity has been documented [26, 30]. FIB-4 were associated with the severity of the OSA. Considering the role of chronic intermittent hypoxia in the progression of metabolic liver disease [24, 33], it can be hypothesized that the indirect scores of liver disease worsen with greater number and severity of desaturations. It has been described that in pediatric NAFLD the presence of OSA is significantly associated with the presence of NASH and fibrosis, and that the severity of desaturations was related to the NASH scores and the extent of fibrosis, regardless of the degree of obesity, adiposity abdominal, metabolic syndrome and insulin resistance [42]. In children and adolescents with obesity, the degree of histologically shown fibrosis was more severe in subjects with NAFLD and OSA than in patients without respiratory syndrome. In addition, the severity and duration of nocturnal hypoxemia were associated with the progression of the histological features of NAFLD and the elevation of transaminases [43]. Finally, a direct correlation between the histological elements of NASH (degree of steatosis, degree of lobular inflammation, NAS scores and stage of fibrosis) and the presence and severity of OSA has been shown [44].

Consequently, non-invasive biomarkers and scores could become a screening tool to identify patients living with obesity and OSA to be referred to liver biopsy and therefore to prevent NAFLD progression. Indeed, could be useful to recognize those patients with OSA and higher risk to develop severe fibrosis. With this aim we focused on polysomnographic parameters which describe OSA severity. To investigate the role of different polysomnographic parameters as independent predictors of liver fibrosis, we computed a multiple regression model with FIB-4 as the dependent variable. In this model, TG, sex, meanSaO2\%, BMI and HOMA were the only variables independently correlated with FIB-4 in 334 patients with obesity. It is well known how TG and insulin resistance contribute to pathogenesis to NAFLD [22], that NAFLD prevalence and severity are greater in male and that there is a strong association between NAFLD and BMI [1, 19]. Conversely, we described an inverse correlation between the two variables FIB-4 and BMI. This may be explained by the so-called "obesity paradox" where patients with obesity and cirrhosis have lower mortality than normal weight patients with cirrhosis [45]. Interestingly, we demonstrated that the meanSaO2\% and not AHI, among polysomnographic parameters, is an independent determinant of FIB-4. A possible explanation may be that AHI quantifies the number of apnea episodes during the polysomnographic recording, without considering severity. The average saturation, although related to AHI values, 
considers the severity of desaturation events and is determined not only by the number, but also by the duration and depth of the desaturations. Thus, meanSaO2\% may represent a more precise parameter to describe and correlate OSA chronic intermittent hypoxia to liver fibrosis scores.

Our study has some limitations. Firstly, we enrolled patients affected by severe obesity in a center where bariatric surgery is the main suggested treatment. This may explain the high prevalence of obesity-related comorbidities. Furthermore, the distribution of patients in the OSA classes is not balanced. The great sample size of subjects with severe OSA is explained by the fact that polysomnography is a survey that requires a not negligible commitment of time and health resources and is therefore prescribed only for symptomatic patients or with high scores on predictive tests for OSA. Indeed, electroencephalogram is lacking, nevertheless the polysomnographic analysis was performed according to the most recent AASM criteria [37] and, thus, an appropriate method. The lack of ethnic diversity is a limitation to generalizability of our findings. Finally, we did not perform liver biopsy, but this invasive procedure cannot be extended to all patients with obesity as a screening method [26-28]. Therefore, in accordance with recent guidelines, we used fibrosis scores to stratify NAFLD patients and only patients with high scores of fibrosis undergo histological confirmations with a liver biopsy $[2,3]$.

In conclusion, our study shows that the presence and severity of the OSA, defined by international guidelines based on the AHI, is associated with an increased in TG values and insulin resistance, suggesting an underling common pathogenetic mechanism between OSA and NAFLD. Furthermore, we demonstrated the association of fibrosis, and OSA severity in adult patients with severe obesity, confirming previous studies $[32,33,44]$. A novelty finding is that mean $\mathrm{SaO} 2 \%$ is independently related to FIB-4. Therefore, meanSaO2\% could play a role in recognizing patients with obesity and OSA with higher risk of developing advanced fibrosis and, thus, to undergo further investigation.

\section{What is already known on this subject?}

- OSA correlates with NAFLD and OSA patients should be screened for the presence and severity of NAFLD.

- It is unclear the association between polysomnographic parameters and markers/scores of fibrosis.

\section{What this study adds?}

- meanSaO2 is independently related to FIB-4, suggesting a clinical implication for recognizing patients with obesity and OSA and a higher risk of developing advanced fibrosis.
Acknowledgements We extend our gratitude to Dr. Anna Belligoli, Dr. Valentina Silvestrin, Dr. Jenni Turra, Dr. Angelo Di Vincenzo, Dr. Martina Ferrata for their contribution to the collection of clinical data.

Author contributions SB, RS and LB: Conceptualized the study, the methodology and prepared the original draft; SB, RS, FF, FD, CD: Participated in the data curation and the statistical analyses; SB, CDP, RF, RS, LB: Provided clinical data and samples; RV and LB: Supervised the study, reviewed and edited the manuscript.

Funding Open access funding provided by Università degli Studi di Padova within the CRUI-CARE Agreement.

\section{Declarations}

Conflict of interest Pr. LB reports personal fees from Novo Nordisk and from Bruno Farmaceutici, outside the submitted work. Dr. SB, RS, CDP, RF, FF, CD, FD, RV declare no conflict of interest.

Ethical approval All subjects gave written informed consent in accordance with the Declaration of Helsinki. The protocol was approved by the 'Padua Ethical Committee for Clinical Research' (2892P, $10 / 06 / 2013)$. This statement was also included in methods section.

Informed consent All subjects gave written informed consent in accordance with the Declaration of Helsinki.

Open Access This article is licensed under a Creative Commons Attribution 4.0 International License, which permits use, sharing, adaptation, distribution and reproduction in any medium or format, as long as you give appropriate credit to the original author(s) and the source, provide a link to the Creative Commons licence, and indicate if changes were made. The images or other third party material in this article are included in the article's Creative Commons licence, unless indicated otherwise in a credit line to the material. If material is not included in the article's Creative Commons licence and your intended use is not permitted by statutory regulation or exceeds the permitted use, you will need to obtain permission directly from the copyright holder. To view a copy of this licence, visit http://creativecommons.org/licenses/by/4.0/.

\section{References}

1. Younossi ZM, Koenig AB, Abdelatif D, Fazel Y, Henry L, Wymer $M$ (2016) Global epidemiology of nonalcoholic fatty liver disease-meta-analytic assessment of prevalence, incidence, and outcomes. Hepatology 64:73-84. https://doi.org/10.1002/hep.28431

2. European Association for the Study of the Liver (EASL); European Association for the Study of Diabetes (EASD); European Association for the Study of Obesity (EASO) (2016) EASLEASD-EASO: clinical practice guidelines for the management of non-alcoholic fatty liver disease. J Hepatol 64(6):1388-1402. https://doi.org/10.1016/j.jhep.2015.11.004.

3. Chalasani N, Younossi Z, Lavine JE, Charlton M, Cusi K, Rinella $M$ et al (2018) The diagnosis and management of nonalcoholic fatty liver disease: practice guidance from the American Association for the study of liver diseases. Hepatology 67(1):328-357. https://doi.org/10.1002/hep.29367

4. Destors M, Tamisier R, Galerneau LM, Lévy P, Pepin JL (2017) Pathophysiology of obstructive sleep apnea syndrome and its cardiometabolic consequences. Presse Med 46(4):395-403 
5. Hopps E, Caimi G (2015) Obstructive sleep apnea syndrome: links between pathophysiology and cardiovascular complications. Clin Invest Med 38(6):E362-E370

6. Peppard PE, Young T, Barnet JH et al (2013) Increased prevalence of sleep disordered breathing in adults. Am J Epidemiol 177:1006-1014

7. Watanabe M, Risi R, Camajani E, Contini S, Persichetti A, Tuccinardi D et al (2020) Baseline HOMA IR and circulating FGF21 levels predict NAFLD improvement in patients undergoing a low carbohydrate dietary intervention for weight loss: a prospective observational pilot study. Nutrients 12(7):2141. https://doi.org/ $10.3390 /$ nu 12072141

8. Sateia MJ (2014) International classification of sleep disordersthird edition: highlights and modifications. Chest 146:1387-1394

9. Lévy P, Kohler M, McNicholas WT, Barbé F, McEvoy RD, Somers VK et al (2015) Obstructive sleep apnoea syndrome. Nat Rev Dis Primers 1:15015

10. Bozkurt NC, Beysel S, Karbek B, Unsal İO, Cakir E, Delibasi T (2016) Visceral obesity mediates the association between metabolic syndrome and obstructive sleep apnea syndrome. Metab Syndr Relat Disord 14(4):217-221

11. Lévy P, Bonsignore MR, Eckel J (2009) Sleep, sleep-disordered breathing and metabolic consequences. Eur Respir J 34:243260. https://doi.org/10.1183/09031936.00166808

12. Ursavas A, Ilcol YO, Nalci N, Karadag M, Ege E (2010) Ghrelin, leptin, adiponectin, and resistin levels in sleep apnea syndrome: role of obesity. Ann Thorac Med 5(3):161-165

13. Söğüt A, Açıkgöz Ş, Uzun L, Uğur MB, Altın R, Dağlı E et al (2016) Leptin levels in children with obstructive sleep apnea syndrome. Tuberk Toraks 64(4):283-288

14. Lacedonia D, Nigro E, Matera MG, Scudiero O, Monaco ML, Polito R et al (2016) Evaluation of adiponectin profile in Italian patients affected by obstructive sleep apnea syndrome. Pulm Pharmacol Ther 40:104-108

15. Trakada G, Steiropoulos P, Nena E, Gkioka T, Kouliatsis G, Pataka A et al (2009) Plasma visfatin levels in severe obstructive sleep apnea-hypopnea syndrome. Sleep Breath 13(4):349-355

16. Reilly SM, Saltiel AR (2017) Adapting to obesity with adipose tissue inflammation. Nat Rev Endocrinol 13(11):633-643

17. Jin F, Liu J, Zhang X, Cai W, Zhang Y, Zhang W et al (2017) Effect of continuous positive airway pressure therapy on inflammatory cytokines and atherosclerosis in patients with obstructive sleep apnea syndrome. Mol Med Rep 16(5):6334-6339

18. Busetto L, Bettini S, Fabris R, Serra R, Dal Pra C, Maffei P et al (2020) Obesity and COVID-19: an Italian snapshot. Obesity (Silver Spring) 28(9):1600-1605

19. Bedogni G, Miglioli L, Masutti F, Tiribelli C, Marchesini G, Bellentani S (2005) Prevalence of and risk factors for nonalcoholic fatty liver disease: the Dionysos nutrition and liver study. Hepatology 42:44-52

20. Polyzos SA, Kountouras J, Mantzoros CS (2016) Adipokines in nonalcoholic fatty liver disease. Metabolism 65(8):1062-1079

21. Aron-Wisnewsky J, Minville C, Tordjman J, Levy P, Bouillot JL, Basdevant A et al (2012) Chronic intermittent hypoxia is a major trigger for non-alcoholic fatty liver disease in morbid obese. J Hepatol 56:225-233

22. Buzzetti E, Pinzani M, Tsochatzis EA (2016) The multiplehit pathogenesis of non-alcoholic fatty liver disease (NAFLD). Metabolism 65(8):1038-1048. https://doi.org/10.1016/j.metab ol.2015.12.012

23. Simões ICM, Fontes A, Pinton P, Zischka H, Wieckowski MR (2018) Mitochondria in non-alcoholic fatty liver disease. Int J Biochem Cell Biol 95:93-99. https://doi.org/10.1016/j.biocel. 2017.12.019

24. Savransky V, Nanayakkara A, Vivero A, Li J, Bevans S, Smith PL et al (2007) Chronic intermittent hypoxia predisposes to liver injury. Hepatology 45(4):1007-1013. https://doi.org/10. 1002/hep. 21593

25. Ekstedt M, Hagström H, Nasr P, Fredrikson M, Stål P, Kechagias $\mathrm{S}$ et al (2015) Fibrosis stage is the strongest predictor for disease-specific mortality in NAFLD after up to 33 years of follow-up. Hepatology 61(5):1547-1554

26. Cleveland E, Bandy A, VanWagner LB (2018) Diagnostic challenges of nonalcoholic fatty liver disease/nonalcoholic steatohepatitis. Clinical Liver Disease 11:98-104

27. Tapper EB, Lok AS (2017) Use of liver imaging and biopsy in clinical practice. N Engl J Med 377:756-768. https://doi.org/ 10.1056/NEJMra1610570

28. Ratziu V, Charlotte F, Heurtier A, Gombert S, Giral P, Bruckert E, LIDO Study Group et al (2005) Sampling variability of liver biopsy in nonalcoholic fatty liver disease. Gastroenterology 128:1898-1906

29. Kim D, Kim WR, Kim HJ, Therneau TM (2013) Association between noninvasive fibrosis markers and mortality among adults with nonalcoholic fatty liver disease in the United States. Hepatology 57(4):1357-1365. https://doi.org/10.1002/hep. 26156

30. Shah A, Lydecker A, Murray K, Tetri B, Contos M, Sanyal A et al (2009) Use of the Fib4 index for non-invasive evaluation of fibrosis in nonalcoholic fatty liver disease. Clin Gastroenterol Hepatol 7(10):1104-1112. https://doi.org/10.1016/j.cgh.2009. 05.033

31. Yilmaz Y, Yonal O, Kurt R, Bayrak M, Aktas B, Ozdogan O (2011) Noninvasive assessment of liver fibrosis with the aspartate transaminase to platelet ratio index (APRI): usefulness in patients with chronic liver disease. Hepat Mon 11(2):103-106

32. Musso G, Cassader M, Olivetti C, Rosina F, Carbone G, Gambino R (2013) Association of obstructive sleep apnoea with the presence and severity of non-alcoholic fatty liver disease. A systematic review and meta-analysis. Obesity Rev 14(5):417-431

33. Paschetta E, Belci P, Alisi A, Liccardo D, Cutrera R, Musso G et al (2015) OSAS-related inflammatory mechanisms of liver injury in nonalcoholic fatty liver disease. Mediators Inflamm 2015:1-10

34. Friedewald WT, Levy RI, Fredrickson DS (1972) Estimation of the concentration of low-density lipoprotein cholesterol in plasma, without use of the preparative ultracentrifuge. Clin Chem 18(6):499-502

35. Siekmann L, Bonora R, Burtis CA, Ceriotti F, Clerc-Renaud P, Ferard G et al (2002) IFCC primary reference procedures for the measurement of catalytic activity concentrations of enzymes at 37 degrees C. International Federation of Clinical Chemistry and Laboratory Medicine. Part 7. Certification of four reference materials for the determination of enzymatic activity of gamma-glutamyltransferase, lactate dehydrogenase, alanine aminotransferase and creatine kinase accord. Clin Chem Lab Med 40(7):739-745

36. Matthews DR, Hosker JP, Rudenski AS, Naylor BA, Treacher DF, Turner RC (1985) Homeostasis model assessment: insulin resistance and $\beta$-cell function from fasting plasma glucose and insulin concentrations in man. Diabetologia 28:412-419

37. Berry RB, Brooks R, Gamaldo C, Harding SM, Lloyd RM, Quan SF et al (2017) AASM scoring manual updates for 2017 (Version 2.4). J Clin Sleep Med 13(5):665

38. Mach F, Baigent C, Catapano AL, Koskinas KC, Casula M, Badimon L et al (2020) 2019 ESC/EAS Guidelines for the management of dyslipidaemias: lipid modification to reduce cardiovascular risk. Eur Heart J 41:111-188

39. American Diabetes Association (2017) Classification and diagnosis of diabetes. Diabetes Care 40:S11-S24 
40. Young T, Finn L, Peppard PE, Szklo-Coxe M, Austin D, Nieto J et al (2008) Sleep disordered breathing and mortality: eighteen-year follow-up of the Wisconsin sleep cohort. Sleep 31(8):1071-1078. https://doi.org/10.5665/sleep/31.8.1071

41. Feng Y, Zhang Z, Dong ZZ (2015) Effects of continuous positive airway pressure therapy on glycaemic control, insulin sensitivity and body mass index in patients with obstructive sleep apnoea and type 2 diabetes: a systematic review and metaanalysis. NPJ Primary Care Respir Med 25:15005. https://doi. org/10.1038/npjpcrm.2015.5

42. Nobili V, Cutrera R, Liccardo D, Pavone M, Devito R, Giorgio $\mathrm{V}$ et al (2014) Obstructive sleep apnea syndrome affects liver histology and inflammatory cell activation in pediatric nonalcoholic fatty liver disease, regardless of obesity/insulin resistance. Am J Respir Crit Care Med. https://doi.org/10.1164/rccm. 201307-13390C

43. Sundaram SS, Sokol RJ, Capocelli KE, Pan Z, Sullivan JS, Robbins $\mathrm{K}$ et al (2014) Obstructive sleep apnea and hypoxemia are associated with advanced liver histology in pediatric nonalcoholic fatty liver disease. J Pediatr 164(4):699-706.e1

44. Corey KE, Misdraji J, Gelrud L, King LY, Zheng H, Malhotra A et al (2015) Obstructive sleep apnea is associated with nonalcoholic steatohepatitis and advanced liver histology. Dig Dis Sci 60(8):2523-2528

45. Karagozian R, Bhardwaj G, Wakefield DB, Baffy G (2016) Obesity paradox in advanced liver disease: obesity is associated with lower mortality in hospitalized patients with cirrhosis. Liver Int 36(10):1450-1456. https://doi.org/10.1111/liv.13137

Publisher's Note Springer Nature remains neutral with regard to jurisdictional claims in published maps and institutional affiliations. 